\title{
Production and Reliability Oriented SOFC Cell and Stack Design
}

\author{
M. Hauth ${ }^{\mathrm{a}}$, V. Lawlor ${ }^{\mathrm{a}}$, P. Cartellieri ${ }^{\mathrm{a}}$, C. Zechmeister ${ }^{\mathrm{b}}$, S. Wolff ${ }^{\mathrm{b}}$, C. Bucher $^{\mathrm{b}}$

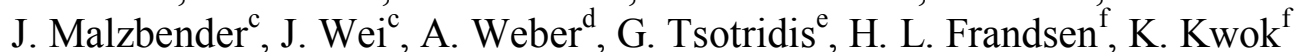

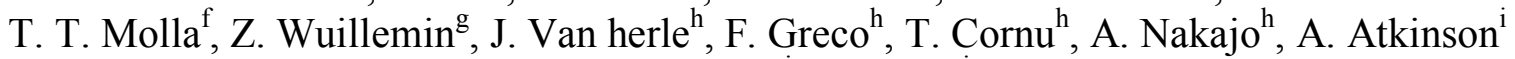 \\ L. Vandeperre ${ }^{i}, X$. Wang ${ }^{i}$ \\ ${ }^{a}$ AVL List GmbH, Hans-List-Platz 1, 8020 Graz, Austria \\ ${ }^{\mathrm{b}}$ Dynardo Austria GmbH, Wagenseilgasse 14, 1120 Wien, Austria \\ ${ }^{c}$ Forschungszentrum Jülich GmbH, Institute for Energy and Climate Research, IEK-2 \\ 52425 Jülich, Germany \\ ${ }^{\mathrm{d}}$ Institute for Applied Materials (IAM-WET), Karlsruher Institut für Technologie (KIT) \\ Adenauerring 20b, 76131 Karlsruhe, Germany \\ ${ }^{\mathrm{e}}$ European Commission, Joint Research Centre, Directorate C: Energy, Transport and \\ Climate, Petten, The Netherlands \\ ${ }^{\mathrm{f}}$ DTU, Frederiksborgvej 399, 4000 Roskilde, Denmark \\ ${ }^{g}$ SOLIDpower-HTceramix, 26 av. des Sports, 1400 Yverdon-les-Bains, Switzerland \\ ${ }^{\mathrm{h}}$ EPFL Valais, rue de 1'Industrie 17, 1951 Sion, Switzerland \\ ${ }^{\mathrm{i}}$ Imperial College, London, SW7 2AZ, UK
}

This paper presents an innovative development methodology for a production and reliability oriented SOFC cell and stack design aiming at improving the stacks robustness, manufacturability, efficiency and cost. Multi-physics models allowed a probabilistic approach to consider statistical variations in production, material and operating parameters for the optimization phase. A methodology for 3D description of spatial distribution of material properties based on a random field models was developed and validated by experiments. Homogenized material models on multiple levels of the SOFC stack were established. The probabilistic models were related to the experimentally obtained properties of base materials to establish a statistical relationship between the material properties and the most relevant load effects. Software algorithms for meta models that allow the detection of relationships between input and output parameters and to perform a sensitivity analysis were developed and implemented. The capabilities of the methodology are illustrated on two practical cases.

\section{Introduction}

This paper reports the results from the European project PROSOFC. Within the project an innovative methodology for a production and reliability oriented SOFC cell and stack design is developed. In particular the PROSOFC project aims at improving the robustness, manufacturability, efficiency and cost of SOLIDpower state-of-the-art SOFC stacks so as to reach market entry requirements. The key issues are the mechanical robustness of SOFCs, and the delicate interplay between cell properties, stack design, and operating conditions of the SOFC stack. 
The novelty of the project lies in combining state of the art methodologies for costoptimal reliability-based design (COPRD) with actual production optimization. To achieve the COPRD beyond state of the art, multi-physical modeling concepts were developed and validated for significantly improved understanding of the production and operation of SOFC stacks. The most critical failure modes for stack operation were identified in order to align the experimental investigations to generate suitable data for modelling these failures. The multi-physics model allows a probabilistic approach to consider statistical variations in production, material and operating parameters for the optimization phase. The project provides a methodology for 3D description of spatial distribution of material properties based on a random field models. The key for the whole methodology are validating experiments, homogenized models on multiple levels of the SOFC system (cell, interconnect, sealings) and introduction of extensive test programs specified by the COPRD methodology. The probabilistic models were related to the experimentally obtained properties of base materials to establish a statistical relationship between the material properties and the most relevant load effects with respect to potential damage/failure modes. Software algorithms for meta models that allow the detection of relationships between input parameters (e.g. materials, loading, etc.) and output parameters (e.g. temperatures, stress, etc.) and to perform a sensitivity analysis were developed and implemented. The capabilities of the methodology for COPRD being developed is illustrated on two practical cases.

\section{Scientific Approach}



Figure 1. PROSOFC work flow. 
The project was structured as shown in Figure 1. Homogenized mechanical material models in WP2 (cell, sealing and interconnect) and electrochemical models in WP3 (cell) were established by experimental investigations to feed the multi-physics stack model in WP4. This multi-physics model was then used for optimization by sensitivity analyses considering the stochastic character of material and electrochemical properties. Material characterization was considering specific failure modes (e.g. loss of contact, cell cracks, breakage of sealing) defined during the reliability development in WP5. The results of the optimization were fed back into the stack design in WP6. WP7 aimed at long term stack testing to produce data for the reliability development and stack design validation. Tested stacks were investigated by post mortem analysis in WP8, which also includes the ongoing cost analysis and cost optimization due to stack design improvements (e.g. reduced failure rate by more robust design). This paper focuses on the core methodology development which is concentrated on the interaction between the WPs 2, 3 and 4.

\section{$\underline{\text { Material Parameter Characterization and Sub-models }}$}

In the multiple simulations of the parameter sensitivities and design changes of the SOFC stack in the COPRD method, it is not feasible to describe discrete geometric material features with the computational power currently available. Thus in WP 2 and 3 the purpose is to bridge the gap between the richly geometrically featured inside of a stack including complex microstructures and the stack models. This can be done by describing the physical behaviour of phenomena occurring at the scale of the representative volume element size for the material effective mechanical, transport and electrochemical properties with simple mathematical expressions, which can easily be integrated in the multi-physics stack model. For instance, cell characteristics is included whereby dependency on porosity, electrode thicknesses are explicitly described in the continuum stack model, although not resolved in detail computationally. Another approach is the use of so-called homogenization to achieve sub-models of e.g. the interconnect to include the mechanical behaviour of this without describing the geometrical features in the overall multi-physics stack model with a significant gain on computational efficiency (1).

\section{Multi-physics Stack Modelling and Testing}

Multi physics models were developed in AVL FIRE and a framework combining gPROMS, FLUENT and ABAQUS for coupled thermo-electrochemical and thermomechanical simulations $(2,3,4)$ and used for the sensitivity analysis using optiSlang. The aim was to establish multi-physics models which could be automatically operated by the optimization software optiSlang. The multi-physics model included the homogenized material and electrochemical effective properties of the characterization experiments. Due to the extensive sensitivity analysis, it was required to develop multi physics models with reasonable runtime. For this reason, the sensitivity analysis was started with simplified 3 cell multi physics models instead of full scale stack models. However, in parallel a full scale multi physics stack model was established to validate the simplified multi physics stack model. For instance, a more realistic temperature distribution along the stack could be simulated with the full scale model since the boundary conditions (e.g. heat loss) and the geometry of the gas manifolds could be considered more realistically. Therefore, it was also possible to distinguish between cells at the center of the stack and 
cells close to the bottom or end plate. This approach allowed to calibrate the simplified multi physics stack model.

To improve the simulation of the temperature field inside the stack a specific close-toreality single cell test was developed to precisely measure the temperature distribution influenced by electrochemical operation and $\mathrm{CH}_{4}$ steam reforming. Locally measured cell temperatures, cell voltages and gas compositions were used to calibrate the CFD simulation model.

\section{$\underline{\text { Stochastic Investigation and Meta Modelling }}$}

Adequate stack design (local geometry and material combination) and operation strategy (load following and thermal cycling) are critical for improving the reliability and long-term durability of SOFC stacks. Due to the complexity of the multi physics analysis cost-optimal probabilistic design cannot be carried out without strongly simplified computational models. Such simplified models, or as they are usually called, meta models, provide an efficient computational concept combining reasonable accuracy with good model performance. Such meta models are established on the basis of input-output relations in which load scenarios and other parameters of real world use are taken as inputs which are systematically varied in a design of experiments (DOE). These support points for the meta model should satisfy the following requirements:

- $\quad$ Explore range of variables by numerical experiments

- Cover range of all variables as uniformly as possible

- Keep number of experiments small

The capabilities of the methodology for COPRD being developed are illustrated on two practical cases.

First, a parameter estimation procedure based on meta models has been developed to measure the elastic, primary and secondary creep model parameters from 4-point bending tests. The goal is to (i) account for the effects of the friction between the sample and the rollers, anticlastic curvature, contact point shift and large deflection, which can affect the accuracy of the measurements, and (ii) measure properties which cannot be obtained using closed-form analytical solutions. This could be achieved by model-based parameter estimation with a 3-D continuum finite-element (FE) model, at the cost of very high running time. The proposed approach is computationally much more efficient. It starts with a sensitivity analysis to generate distributed meta models of the displacement at the inner rollers during loading-unloading cycles and creep measurements simulated by the 3-D FE model. The optimization problem for parameter estimation is then solved using the meta models. The accuracy of the approach was tested using a set of numerical experiments. The experiments on Ni-YSZ samples were then analyzed.

Second, for the stochastic investigation of the thermo-electrochemical behavior of the stack, a multi-physics stack model implemented in gPROMS and Fluent was linked and controlled automatically by optiSlang to perform a sensitivity analysis. The outcome is (i) the identification of the most relevant operating parameters which can have a significant impact on the stack lifetime and (ii) meta models of the efficiency and of the 3-D temperature distribution generated in the view of optimization. The tests presented in the "Results" Section show for instance that the accuracy of the 3-D meta model of the 
temperature distribution is sufficient to identify the minimum of variations in the spatial distribution of the temperature during cycling between full and part load by using sets of operating conditions for optimization.

\section{Material Parameter Characterization and Sub-models}

To make the COPRD method computationally feasible a limited amount of computational volumes / degrees of freedom must be used. Therefore, the physical behaviour of phenomena taking place at the scale of local 3-D microstructural features cannot be described directly, and is described indirectly through statistical or use of submodels / homogenization, i.e. volume averaged effective properties. For instance, the access by the transport of electrons, ions and gas species to the electrocatalytic sites is controlled by the morphology and topology of transport pathways within the heterogeneous electrodes. Further, the strength of the anode support depends on the flaw size (in the range of microns). To describe this, a measured statistical variation of the strength of the anode support is used in the stack models. Also for the various contributions to the area specific resistance, statistical variations are supplied to the stack models. Sub-models are used e.g. to determine the deformational properties (elastic, creep) of the interconnects for a given shape. A finite element model of a repeating unit (size of $\sim 1 \mathrm{~mm}^{3}$ ) of an interconnect is thus used to describe the effective behaviour of it, and in the stack models the interconnect thus appears as a homogeneous anisotropic material, without geometric features.

In the following it is described how the different material characterizations and submodels have been addressed. The work is split into two lines; on mechanical material properties and on electro-chemical properties.

\section{Mechanical Material Modelling and Testing}

Basically, all components in a stack must be characterized mechanically, as they all interact and respond in common to external loads and thermal fields. A number of materials for the SOFC stacks had already been characterized, and these were collected as a base line for simulations at the project beginning. There was however a lack of high temperature measurements, relevant for the operational conditions, in particular. These were consequently addressed in the project.

The variation of the strength (5) and fracture toughness (6) of the cell anode support were measured before and after reduction at operational conditions (temperature, atmosphere). It was found that the strength defining phase of the Ni(O)-YSZ composite are $\mathrm{NiO}$ and YSZ before and after reduction, respectively. The fracture toughness was also found to be higher in the reduced state due to the ductility of the Ni. The fracture toughness was furthermore measured using micro-cantilevers (7) in an indenter, and a simple and useful analysis of this experiment was developed (8). An overview of various measurements techniques and equipment to characterize the mechanical material parameters used was also provided (9).

By modelling the SOFC stack including the effect of creep (time dependent plastic behavior), it was found that the stress and thus the probability of failure increases at the shift of operation (10) (see Figure 2). 


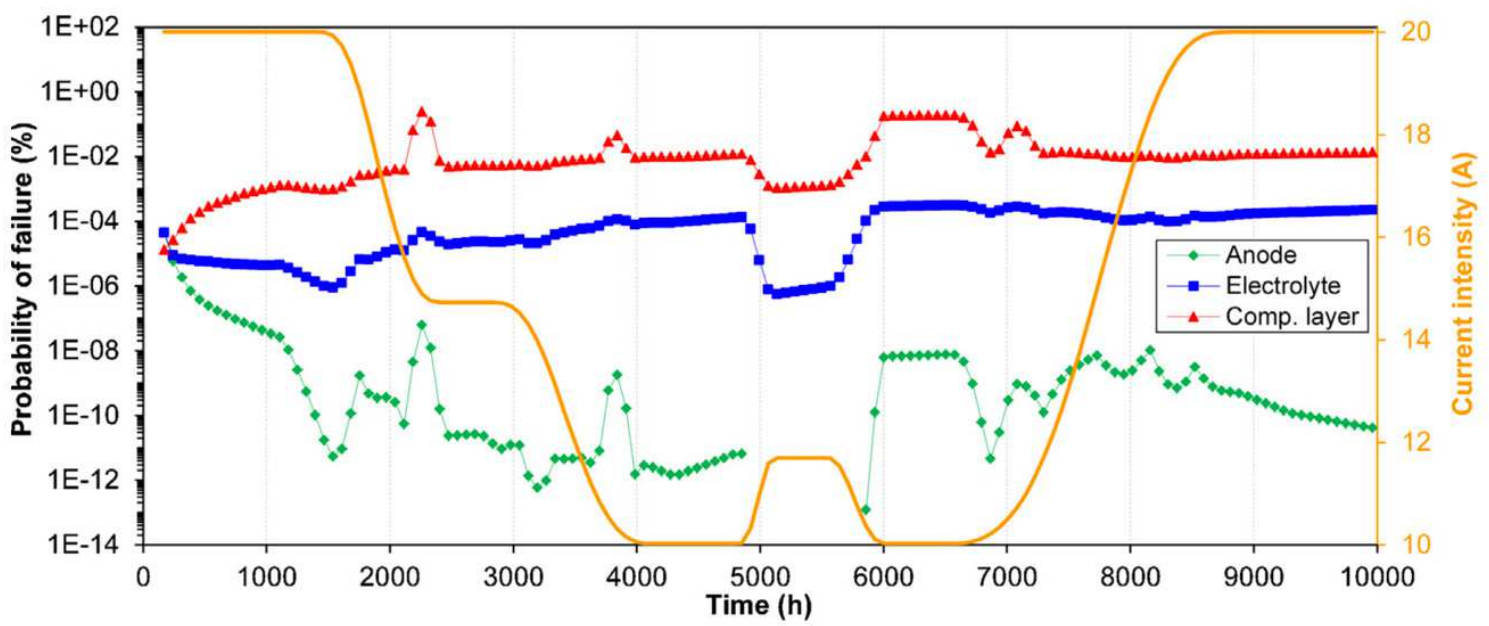

Figure 2. Probability of failure of the anode, electrolyte and compatibility layer for the case of the cell B. The thermal load profile over the SOFC stack lifetime is plotted for reference (10).

Thus, the creep properties of the anode support (11) and metallic interconnects were characterized (12), focusing on both the transient and steady state creep behavior (primary and secondary creep). For the cells creep rates during reduction of the $\mathrm{Ni}(\mathrm{O})$ YSZ supports were found to be orders of magnitude faster than the ones during operation (13) (see Figure 3). This results in stress relaxation of the cell during reduction (papers in preparation).

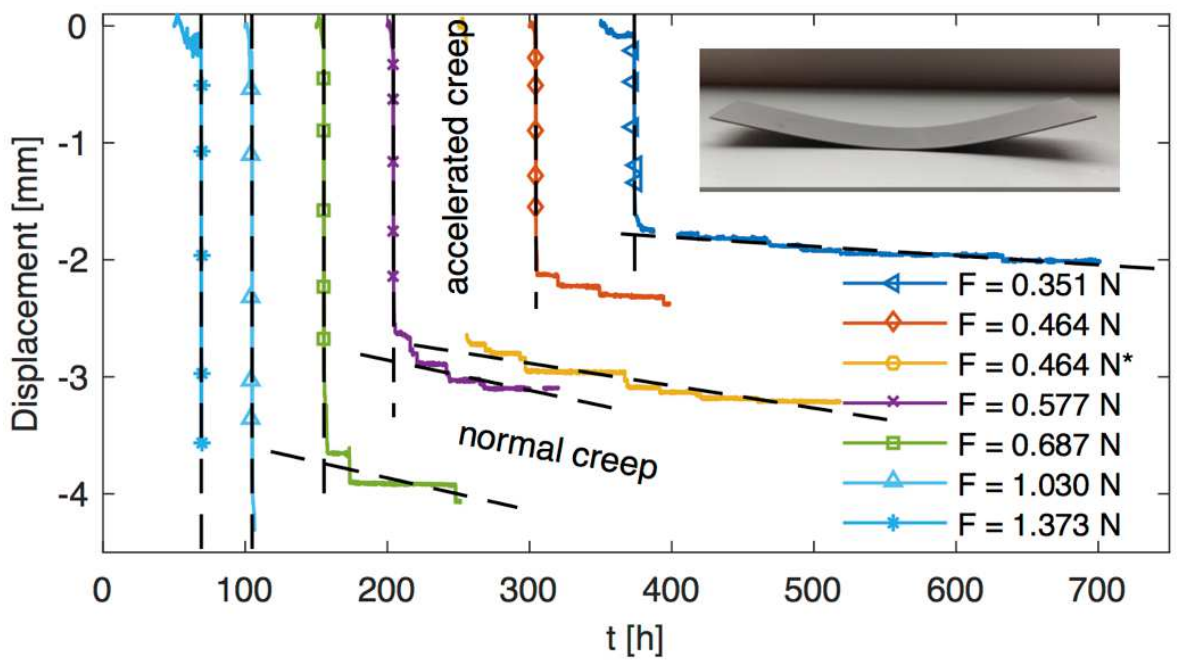

Figure 3. Displacement of the center of beams exposed to different loads in three-point bending and a shift of atmosphere from air to $9 \% \mathrm{H}_{2}$ at $800^{\circ} \mathrm{C}$ (resulting in accelerated creep) (13).

The transient creep of the metallic interconnects was measured using a newly developed rig using a line laser to detect deformations from the loading in-situ a furnace (14). Modelling the creep of the interconnects with all the geometrical features in an entire stack is not computational feasible. Therefore, method for considering this is a homogeneous media with anisotropic material properties equal to those of the original structure was developed (15) (see Figure 4). Similar homogenization approach was used to investigate the creep properties of the Ni-YSZ anode and support, where it was found that YSZ was the rate determining phase through steady state creep (16). 


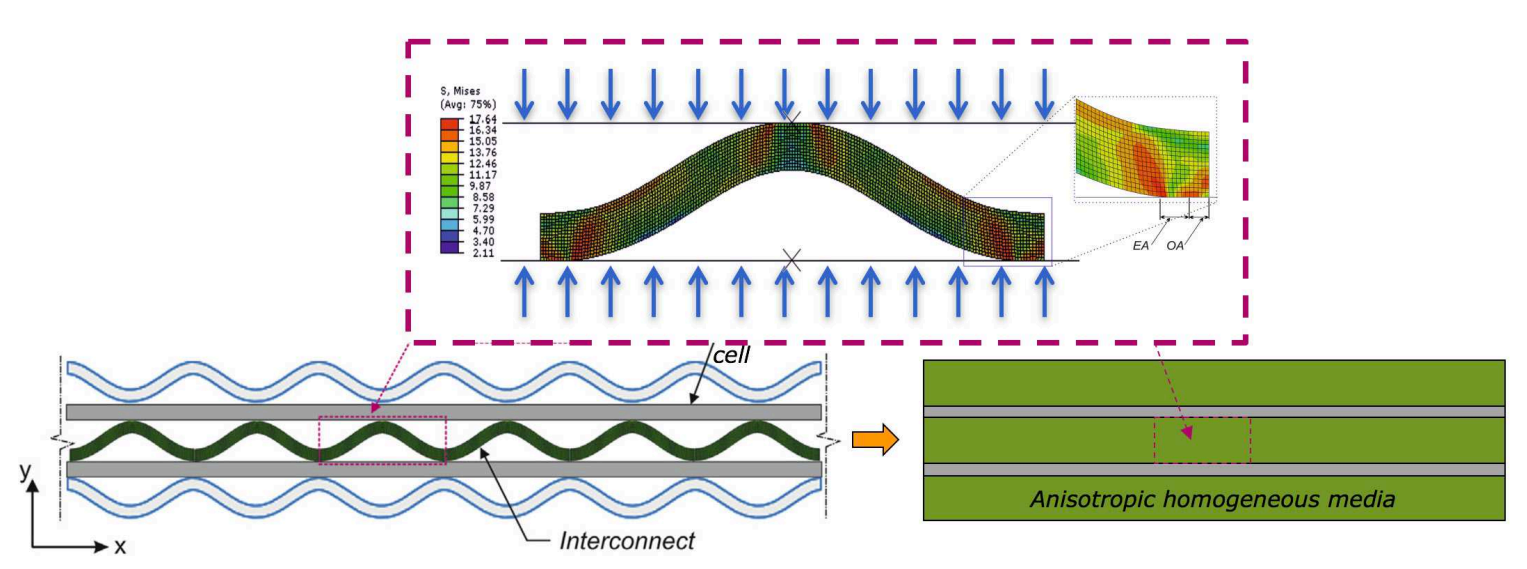

Figure 4. Homogenization of an interconnect by use of sub-modelling (12).

During the entire project time another focus of the work was the mechanical properties of sealant material $(17,18)$, that have verified to be critical for the long term reliability of SOFC stacks (19), where characterization of the fracture stress in bending is based on head-to-head joined specimens (14) and the torsion behavior based on a newly developed elevated temperature torsion tester (13), hence permitting evaluation of the behavior of the sealant materials in different operation relevant loading modes.

\section{Electrochemcial Modelling and Testing}

Physicochemical meaningful equivalent circuit models of the cells are developed based on the IAM-WET approach described in (20). The cells are characterized by electrochemical impedance spectroscopy, the measured impedance spectra are subsequently (i) checked by a Kramers-Kronig test (21), (ii) analyzed by means of their distribution of relaxation times (DRT) (22) and (iii) fitted to an appropriate equivalent circuit model. Previous investigations showed that this approach and the applied model can be adapted to different electrode and cell types (23-25). There are at least 4 electrode processes that can be deconvoluted from the spectra, the gas diffusion in the (anode) substrate $\left(\mathrm{P}_{1 \mathrm{~A}}\right)$, the anode electrochemistry $\left(\mathrm{P}_{2 \mathrm{~A} / 3 \mathrm{~A}}\right)$, either described by $2 \mathrm{RQ}-$ elements $(20)$ or a transmission line model $(26,27)$, the gas diffusion in the cathode $\left(\mathrm{P}_{1 \mathrm{~A}}\right)$ and the oxygen reduction in the mixed conducting cathode $\left(\mathrm{P}_{2 \mathrm{~A}}\right)$, described by a Gerischer impedance (28) in the Adler-Lane-Steele model (29) or even more complex approaches (30). As the different electrode processes can overlap in the frequency domain, they are often not deconvolutable in the DRT of a spectra measured under standard operating conditions. To overcome this (a) operating parameter variations (26) and tests at exceptional operating conditions (31) have to be performed.

The investigated anode supported cells showed processes and characteristic parameter dependencies as expected (see Figure 5). In addition a thermally activated high frequency process with a relaxation frequency of approximately $500 \mathrm{kHz}$ was observed, which does not show any dependency on fuel or oxidant composition. Such process might be attributed to a zirconate layer at the interface to the zirconia electrolyte (32), which have been detected in the SOLIDpower cells by combined transmission electron microscopy (TEM) -energy-dispersive x-ray spectroscopy (EDX) with selected area electron diffraction (SAED) and 3-D focused-ion beam and scanning electron microscopy/energydispersive x-ray spectroscopy (FIB-SEM/EDX, Figure 6). After identification of the 
processes by means of the DRT the spectra can be fitted to an appropriate equivalent circuit model. For stack modeling only the DC-part of the impedance model is required. As described in (33-35), the evaluated model parameters can be implemented in a 0 dimensional stationary cell model, representing an incremental cell unit in a repeat unit (36) or stack model.
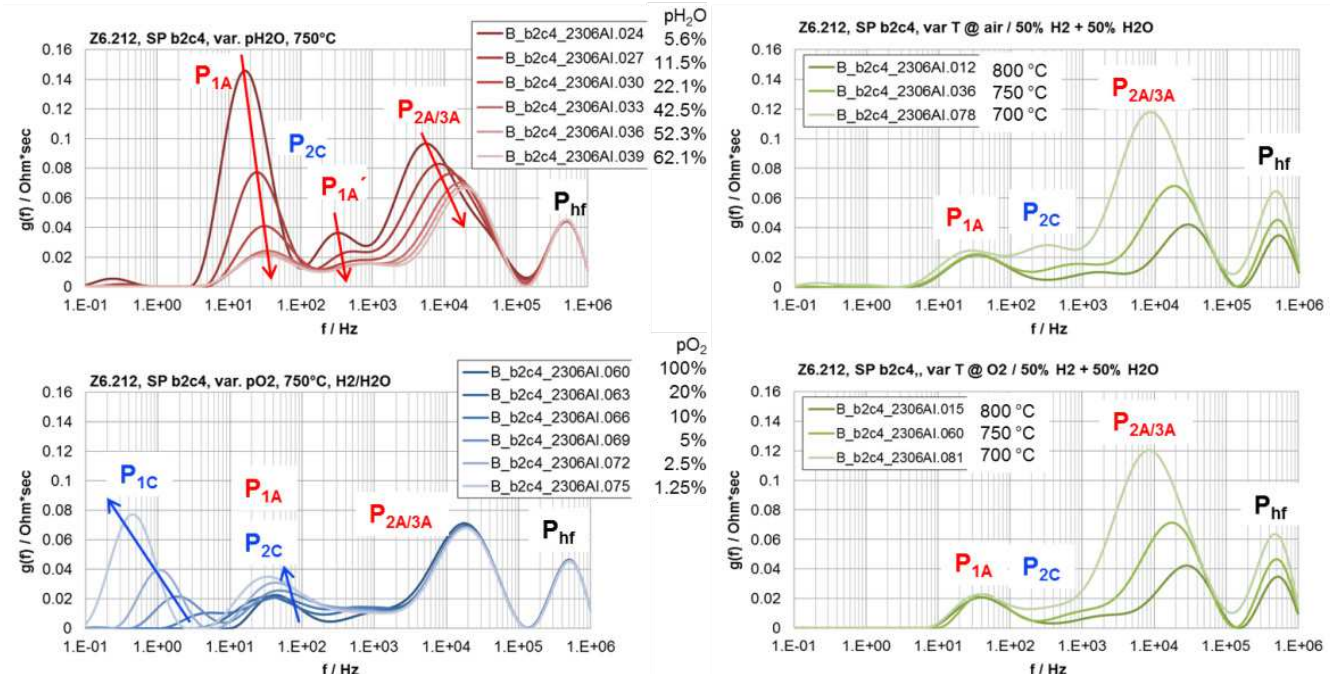

Figure 5. DRTs of impedance spectra measured during different operating parameter variations. Variation of the $\mathrm{pH}_{2} \mathrm{O} / \mathrm{pH}_{2}$-ratio, the $\mathrm{pO}_{2}$ in the oxidant and the temperature shown for oxygen and air as the oxidant

(a)

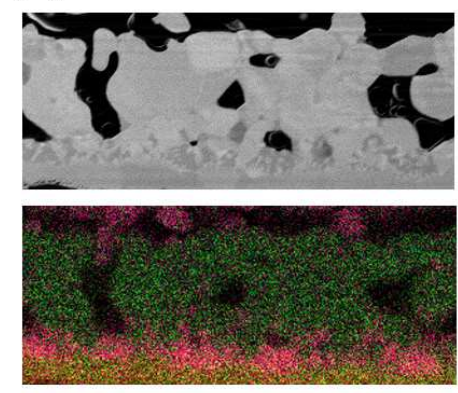

$\square \mathrm{Ni} \square \mathrm{Zr} \square \mathrm{Sr} \square \mathrm{Gd}$

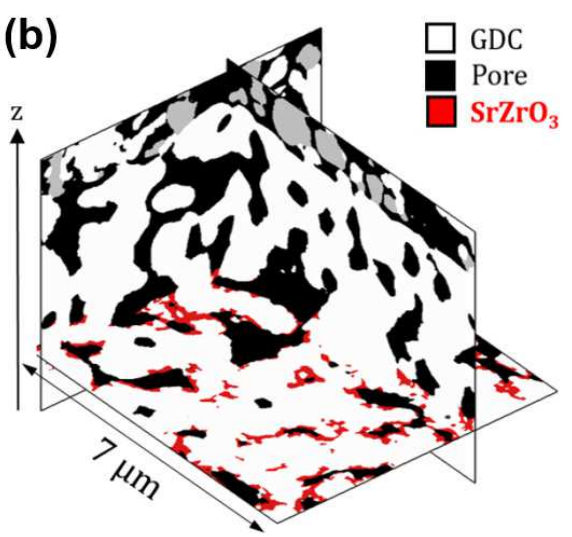

Figure 6. (a) Cross-sections in the 3-D EDX and secondary electron data set showing the accumulation of $\mathrm{Sr}$ at the interface between the YSZ electrolyte and GDC compatibility layer. The phase was confirmed as $\mathrm{SrZrO}_{3}$ by TEM analysis. (b) 3-D view of the $\mathrm{SrZrO}_{3}$ secondary phase after segmentation. The secondary phase was detected already in the pristine cell, but the present figure refers to measurements on a degraded cell.

\section{Multi Physics and Meta Modeling Approach}

\section{$\underline{\text { Stochastic Investigation and Meta Modelling }}$}

An intuitive approach would be to divide each variable into $M$ small intervals and cover all possible combinations of variables $x_{i} ; i=1 \ldots n$. This is a so-called factorial design. It can easily be seen that the total number $N$ of support points equals $N=M^{n}$. This curse of dimensionality (exponential growth with dimension $n$ ) makes factorial 
designs virtually infeasible for complex problems. Therefore Monte Carlo sampling strategies, and later Quasi-Monte Carlo methods such as e.g. Latin Hypercube sampling have beed developed.
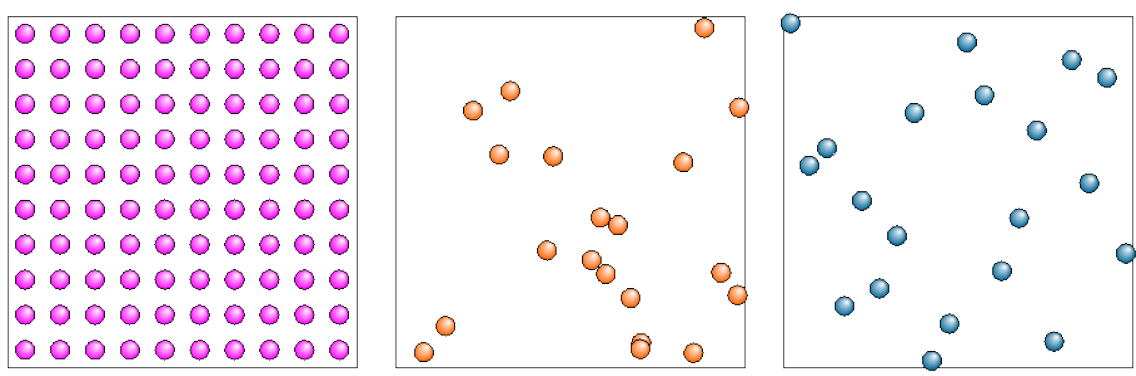

Figure 7. Design of experiments (DOE), left: Factorial design, center: Monte Carlo Sampling, right: Latin Hypercube Sampling

Figure 7 compares factorial design with Monte Carlo sampling and Latin Hypercube sampling. For practical applications, Latin Hypercube sampling is preferred because it provides relatively good uniform coverage of the design space while keeping the number of support points small. The quality of a meta model is descriped by the Coefficient of Prognosis (CoP) which measures the ability of a meta model to correctly predict the response for inputs which have not been used previously to establish the meta model itself. The $\mathrm{CoP}$ is a positive number between 0 an $100 \%$. Additionally, the meta model should be robust enough to be able to cope with a reasonable small fraction of failed simulations (which may arise e.g. from combinations of input parameters for which the solver process does not converge, or a physically sensible solution does not exist).

\section{$\underline{\text { Workflow Sensitivity Analysis }}$}

The sensitivity analysis is performed using meta models, which approximate the actual physical behavior by simple mathematical functions. First steps in the development of COPRD capability for SOFC stack design and operation therefore consisted in (i) identifying the adequate choice of the design variables and initialization sequence for the stack simulations over the range of interest for the sensitivity analysis and (ii) quantifying the accuracy of meta-models for simulating the stack thermo-electrochemical and thermo-mechanical behavior. The next step is the verification of the consistency of the optimization results. At present, two cases have been successfully tested, showcasing the potential for SOFC technology advances provided by the COPRD methodology:

- A 3-D continuum model of the 4-point bending tests has been interfaced with optiSLang. A meta model of the load-displacement relation has been successfully generated and optimizations were performed for "meta model based parameter estimation" of the primary and secondary creep behavior of the Ni-YSZ electrode.

- A metamodel of the 3-D temperature distribution in the stack under a wide range of operating conditions, including variations of power density, gas inlet temperature and fuel composition (degree of pre-reforming), and fuel utilization has been successfully generated. Optimization was then performed to identify the operating conditions to maintain the temperature distribution as steady as 
possible during load following, hence intuitively reduce the risks of uncontrolled failure because of history dependence $(4,37,38,39)$.

\section{Multi-physics Stack Modelling}

A full scale stack simulation based on the real geometry of the SOLIDpower stack was established in AVL FIRE. The aim was to provide a multi-physics simulation model which is able to carry out a detailed analysis of the stack performance which can be used to validate the simplified multi-physics models. The full scale stack model, however, is too large and requires long simulation times which does not allow a reasonably fast sensitivity analysis using the optiSlang software. Especially, the temperature field from the full scale model will be compared with those from the repeating unit of developed in FLUENT/gProms to validate the accuracy of the simplified model. In particular, the local temperature phenomena (e.g. temperature distribution of cells in the stack center vs. cells on top and bottom of stack) were addressed in more detail in the full scale stack model. Also, the impact of the design of the thermal insulation on the stack temperature distribution will be considered. Furthermore, the full scale stack simulation will be aligned with the operating parameters of real stack tests. The scope of the simulation includes not only steady state operation but also the heat-up cycle (see Figure 8) to identify the location of critical temperature gradients within the stack.
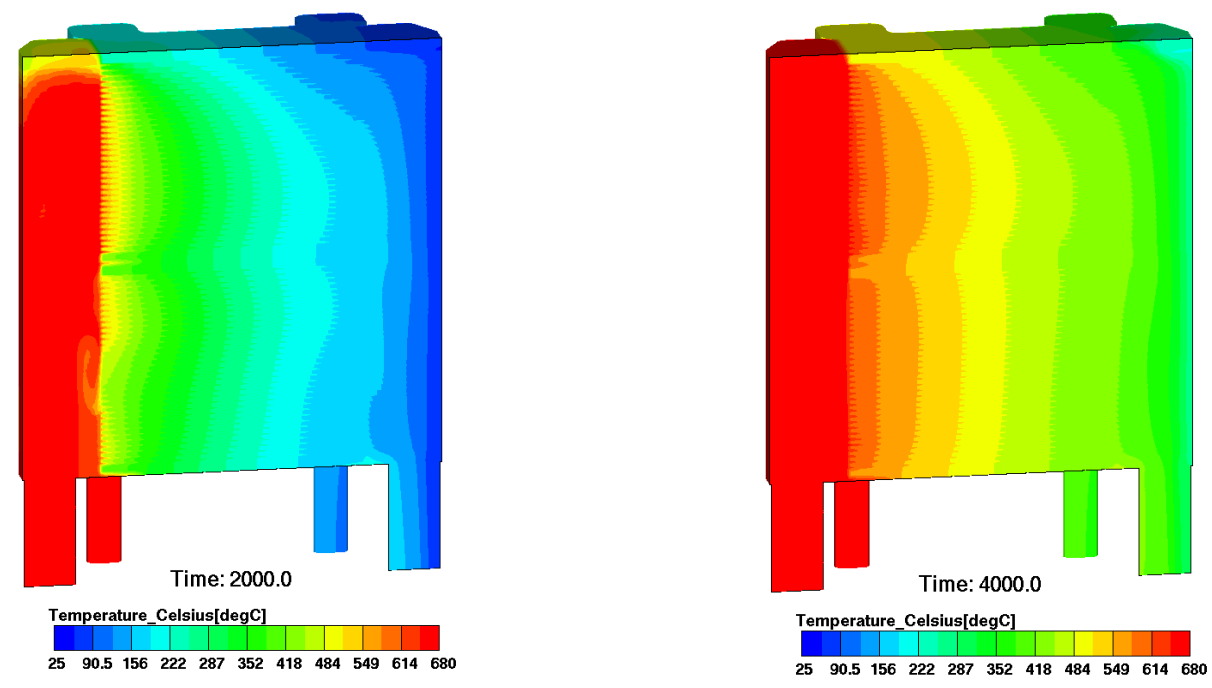

Figure 8. Full scale stack CFD heat up simulation in AVL FIRE.

The full scale stack simulation can be operated in $\mathrm{H}_{2}$ and $\mathrm{CH}_{4}$ steam reforming mode. Especially, the implementation of the reforming kinetics into the full scale stack model was a specific task in this approach. A specifically developed close-to-reality single cell test equipment was experimentally investigated and simulated in CFD. The aim was to measure the temperature field, the cell voltage and the gas composition in $\mathrm{CH}_{4}$ steam reforming operation and use this information to calibrate the AVL FIRE simulation models. These tests provided the kinetic parameters for steam reforming and shift reaction required in the CFD software. The simulated values of the single cell test equipment were in good alignment with the real measured data. The same parameters were then used for the full scale stack simulation. 
The meshing of the geometry in the full scale model (40 million cells) was challenging due to the fact that the mesh has to fulfill certain criteria (conform, hex cells). Out of the full stack model with 66 cell layers first a smaller CFD model ( 3 cell model) was created in order to calibrate parameters for the SOFC module of AVL FIRE CFD code (40) in a faster way.

During the simulation approach the full scale stack model was improved regarding the material parameters and compared with the 3 cell model. The calibration of the full scale stack simulation was based on real operating parameters $\left(\mathrm{H}_{2}\right.$ operation) from SOLIDpower. Therefore, the CV curve of the simulation can be compared with the real measured CV curve from the test. Figure 9 shows the results of the full scale stack (fs) model and the 3 cell model (3c) with old and new material parameters. It can be seen that the model works in good alignment with the real test.

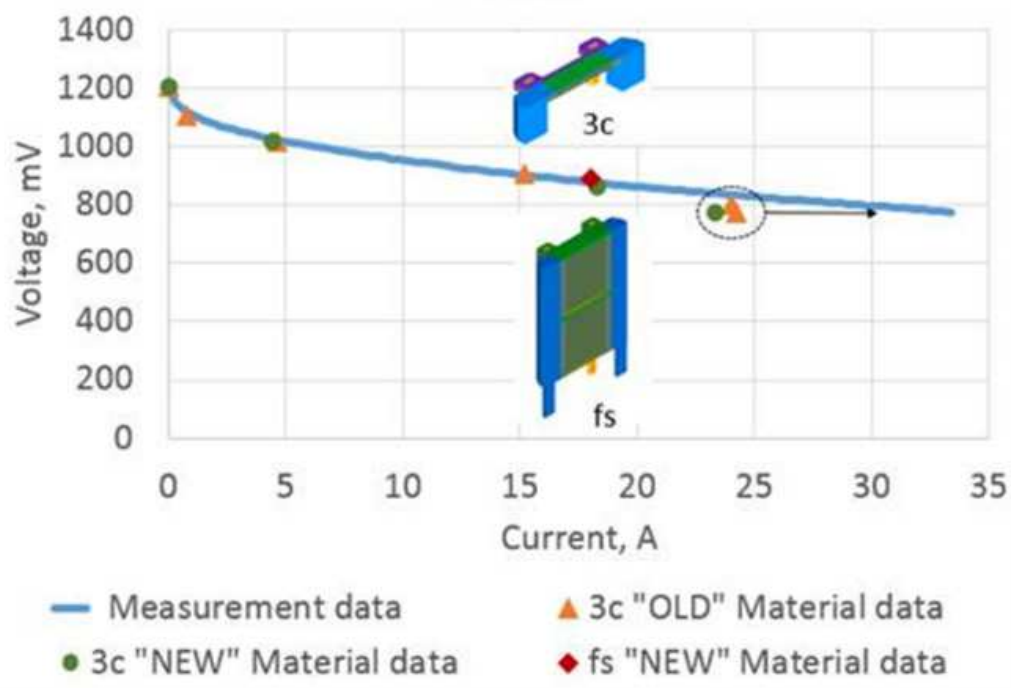

Figure 9. Simulated CV curve of 3 cell (3c) and full scale (fs) stack model with old and new material data in comparison with real measured CV curve (solid curve) from SOLIDpower.

The workflow for the generation of meta models of scalar and 3-D field variables has been established using a modelling framework that model a single repeating unit in a stack. The rationale is the implementation of detailed electrochemistry and material mechanical properties for degradation simulations. To maintain the total runtime within a few days, a single repeating unit is modeled and boundary conditions are modified to simulate the conditions in the middle or close to the end of the stack. In the PROSOFC project, periodic thermal and mechanical conditions were considered for the development of the COPRD methodology. Figure 10 illustrates the combination of the gPROMS local electrochemical model with distributed charge transport and transfer combined with the dusty-gas model and internal steam reforming reaction, fast 1-D stack model for computing the source/sink terms in the active area for 3-D FLUENT simulations of the SOLIDpower stack. 
(a)

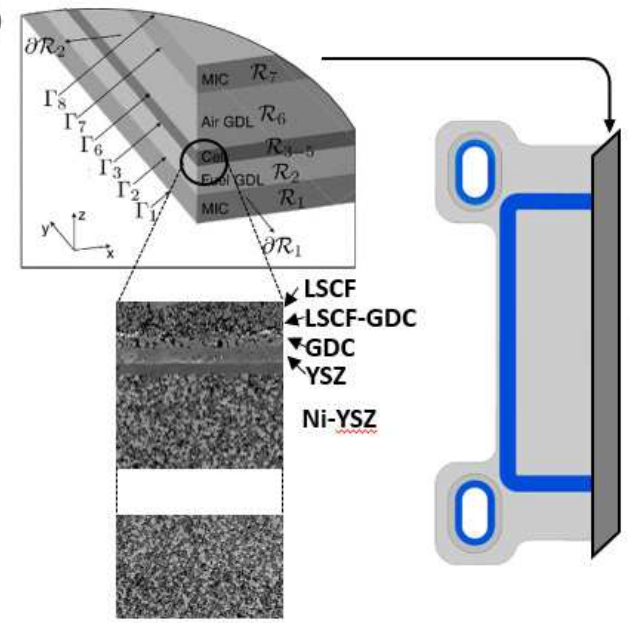

(b)

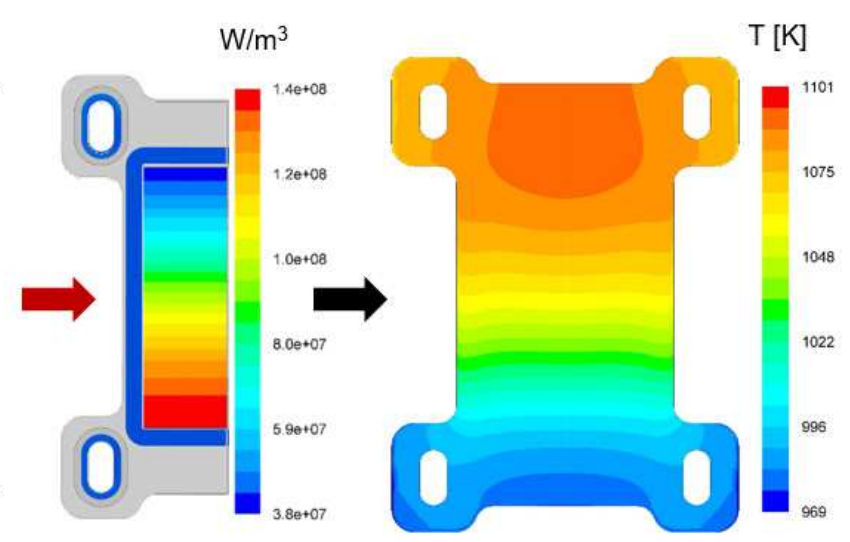

Figure 10. Illustration of the thermo-electrochemical model. (a) Local 1-D electrochemical model combined with 1-D model of the active area. (b) Importation of the source term for 3-D CFD calculations with detailed geometry. (c) Example of a footprint view of the temperature simulated in a repeat element.

In this simplified approach and for the specific SOLIDpower design, the 3-D CFD computation is not required for improved accuracy of the simulated electrochemical performance, but for the simulation of the temperature profile in zones that are not electrochemically active, yet where critical mechanical failure can occur, such as the sealing.The thermo-electrochemical model has been developed mainly for sensitivity analyses and the generation of meta models. Therefore, the electrochemical model has been validated with short-stack data over a wide range of operating conditions, using as input model parameters the effective transport and electrochemical properties measured on 3-D FIB-SEM reconstructions. Figure 11 shows that a satisfactory agreement between simulations and experiment has been achieved over a sufficiently wide range of operating conditions.

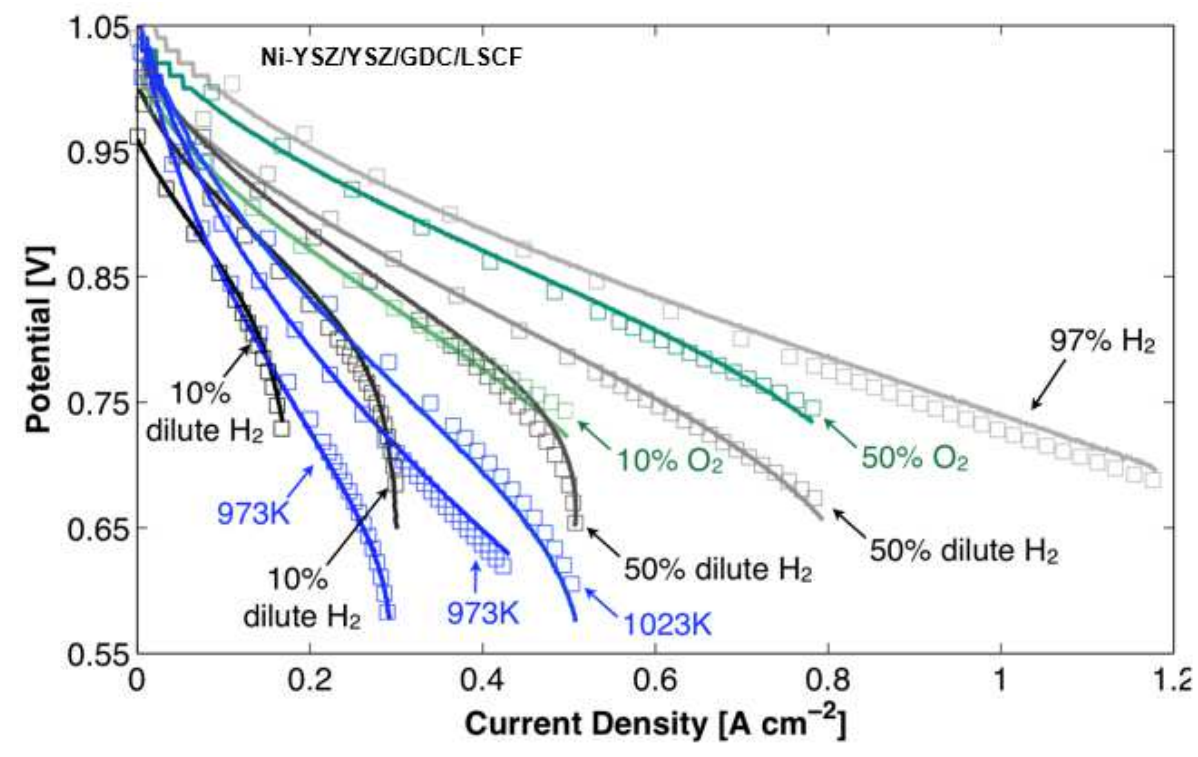

Figure 11. Comparison between I-V characteristics measured on 6-cell short stacks (squares) and simulated (solid lines) with the thermo-electrochemical model. 
The thermo-electrochemical model has been then interfaced with OptiSLang to perform first the sensitivity analyses and then generate the meta-models. Only variations of operating conditions were considered for the test case presented in the "Results" Section. Additionally, variations of the stack geometry/materials were successfully tested within the project, such as thickness of insulation or internal components and aspect ratio of the active area.

Optimization runs with the meta model consisted in the minimization of the local variation of the temperature, when the stack specific power is switched from an arbitrary nominal stack power $\left(0.35 \mathrm{~W} \mathrm{~cm}^{-2}\right)$ to part load conditions $\left(0.225 \mathrm{~W} \mathrm{~cm}^{-2}\right)$. Therefore, the objective function was defined as either the norm or maximum local difference between the temperature at nominal and part load.

A second test case was defined to establish the interaction between OptiSLang and Abaqus. The mechanical properties of ceramic materials are often measured by bending testing because of their brittleness. In the case of four-point bending, intrinsic errors are caused by the friction between the sample and the rollers, anticlastic curvature, contact point shift and large deflection. These effects are not iterated by post-processing with analytical solutions and can affect the accuracy of the measurements. Further, the access to properties such as primary creep parameter require numerical treatment. Therefore, a workflow has been developed to estimate the secondly the primary and secondary creep parameters together with correction of the intrinsic errors in the measurements.

The developed parameters estimation workflow combines: i) a 3-D FE continuum model representing the sample together with the testing fixture of the four-point bending, which provides the simulation responses for a given DoE and ii) the experimental data obtained by four-point bending testing of Ni-YSZ anode samples. A meta model approach has been developed to reduce the computation time of the parameter estimations. The workflow starts with a sensitivity analysis with the FE model to create a distributed meta-model of the four-point bending responses and creep testing. The discretization is here the creep test time. The optimization problem is then solved using the meta model, to identify the parameter values that minimize the discrepancy between the experimental data and the simulated responses.

\section{Results}

\section{$\underline{\text { Sensitivity Analysis of Creep Parameter Estimation }}$}

The accuracy and robustness of the developed meta model based parameter estimation approach was first tested numerically. Artificial experimental data were obtained by simulating the 3-D FE model of the sample and rollers with an arbitrary parameters set. The target properties could be retrieved successfully, regardless of the selection of an optimization starting point over the whole search space.

The experimental data from creep testing of Ni-YSZ samples at $800^{\circ} \mathrm{C}$ in reducing atmosphere and varying loads were fitted with the strain-hardening creep strain rate constitutive law:

$$
\dot{\varepsilon}_{c r}=\left(A(\widetilde{\boldsymbol{q}})^{n}\left[(m+1) \varepsilon_{c r}\right]^{m}\right)^{\frac{1}{m+1}}
$$


This law includes both the primary (parameter $m$ ) and secondary creep regime (parameter $A$ and $n$ ). Figure 12 compares the position of the inner rollers measured during the experiments and simulated by the 3-D FE model with the parameter values determined by the metal model based parameter estimation.

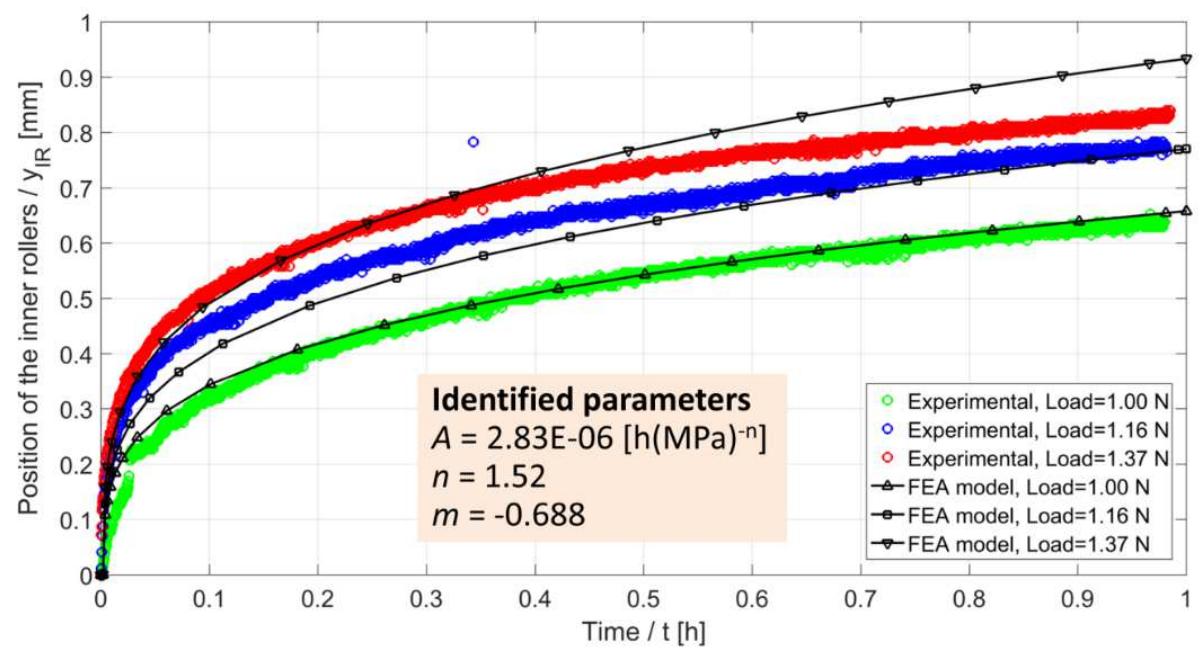

Figure 12. Plot of the creep deformation over time simulated with the FE model with the identified creep parameters (black lines), for three mechanical loads. The experimental creep deformations are plotted for comparison (green, blue and red circles).

\section{$\underline{\text { Sensitivity Analysis of Stack Operation }}$}

A sensitivity analysis was performed with the stack thermo-electrochemical model to generate a 3-D metamodel of the stack temperature distribution. The design of experiment was generated by Latin Hypercube sampling for the following variations of the stack operating conditions:

- Surface specific stack power (nominal: $0.35 \mathrm{~W} \mathrm{~cm}^{-2}$ )

- Maximum stack solid temperature (nominal: $1073 \mathrm{~K}$ )

- Air and fuel inlet temperature (nominal: $973 \mathrm{~K}$ )

- Fraction of methane reformed in the pre-reformer (nominal: 0.25 or 0.99 )

- Fuel utilization (nominal: 0.85)

The analysis of the CoP indicates that the meta model provides an estimate of the temperature profile of good accuracy. This is illustrated in Figure 13part (a), which compares the temperature profile along the symmetry line computed by the thermoelectrochemical and the meta model, and Figure 13part (b) shows that the CoP remain high, which indicates that the meta model provides an estimate of the temperature profile of satisfactory accuracy. Other model uncertainties probably have a greater effect. 


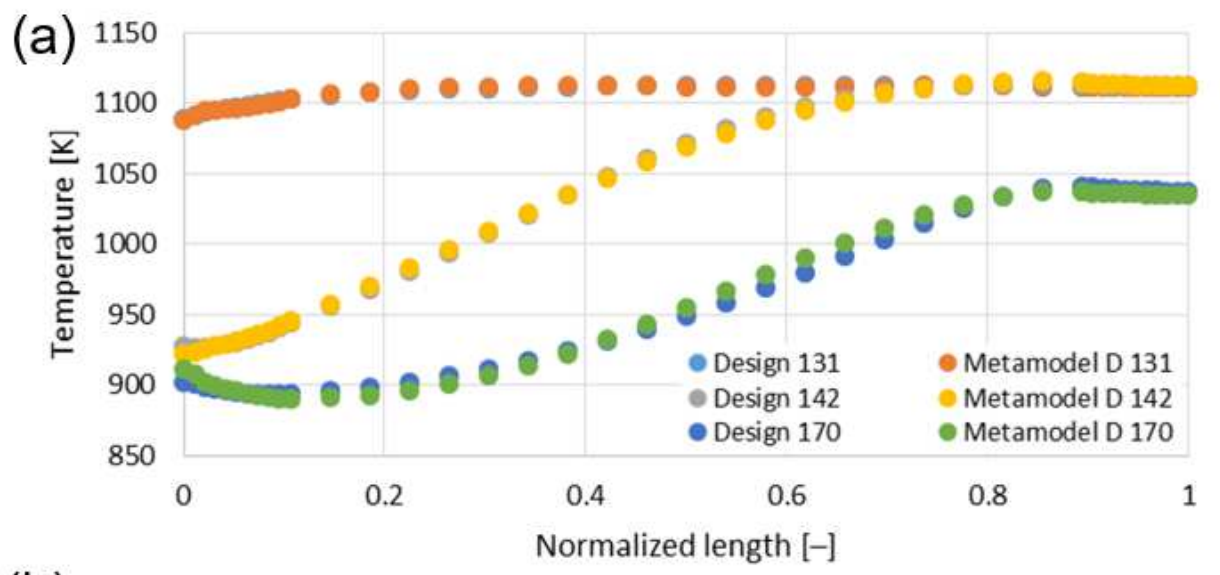

(b)

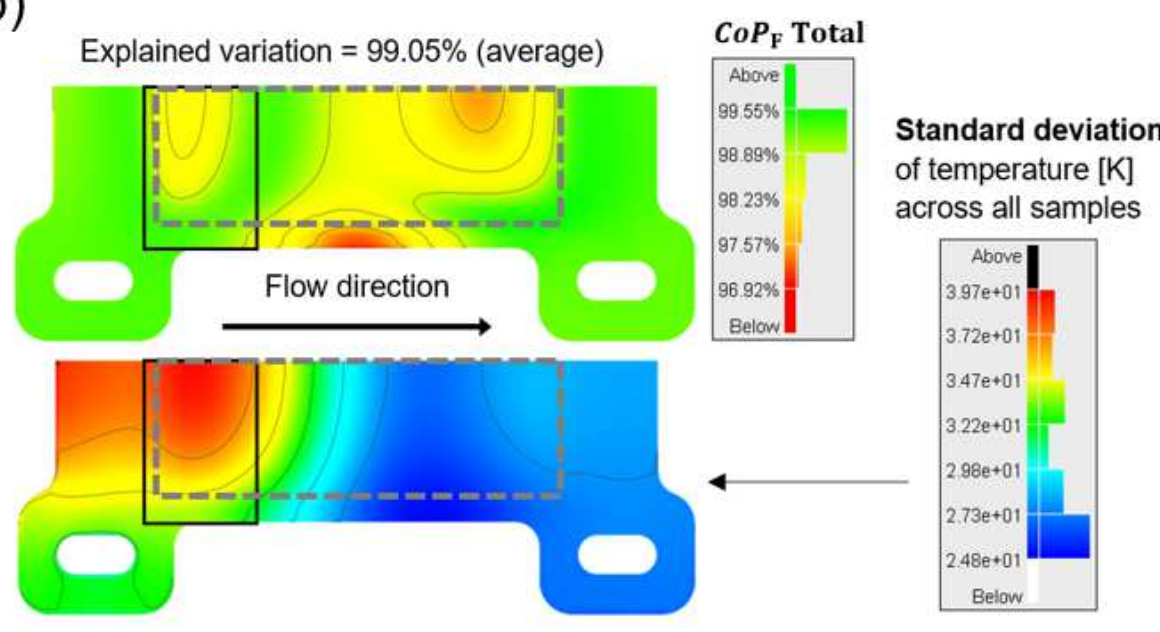

Figure 13. (a) Temperature along the symmetry line for selected simulated samples (designs) compared to the meta model predictions. (b) Top: field of the total coefficient of prognosis of the meta model for the 3-D field of temperature. Bottom: field of the standard deviation of the temperature across all samples. Rectangles in grey-dashed lines show the active area.

Optimization runs were performed with the meta model to minimize variations of temperature upon modification of the stack power density from $0.35 \mathrm{~W} \mathrm{~cm}^{-2}$ (referred to as nominal load) to $0.225 \mathrm{~W} \mathrm{~cm}^{-2}$ (part load). Similar results were obtained using either the norm of the local temperature difference between nominal and part load or the local absolute value, and without region-dependent weighting. Figure 14 provides a summary of the optimization results. The temperature profiles that are shown were computed with the physical thermo-electrochemical model using the operating conditions determined by optimization with the metamodel. By maintaining constant air inlet temperature, fuel utilization and air ratio, local variations of the temperature computed by the metamodel reached 16 and $28 \mathrm{~K}$ when the methane pre-reforming rate is kept constant at 0.99 and 0.25 , respectively. A significant improvement could be achieved by manipulations of the operating conditions at part load. The maximum local variations of the solid temperature could be lowered significantly, below 3 and $6 \mathrm{~K}$ (metamodel calculations).

As a general trend, the optimization leads to a reduction of the air inlet temperature, combined with an increase of the fuel utilization at part load. In practice, fuel utilization higher than 0.9 at part load may result in significant risks of fuel starvation. The 
optimization results indicate that with an additional constraint on the maximum fuel utilization, sub-optimal manipulations of the operating conditions at part load already lead to maximum local variation in the range of $5 \mathrm{~K}$. The minimization of local temperature variations proved more complicated in the case of internal steam methane reforming. Maximum local variations remained in the range of $15 \mathrm{~K}$ after optimization, but could be lowered to $5 \mathrm{~K}$ with enabled adjustments of the pre-reforming rate.

The variations of the temperature computed by the physical model and shown in Figure 14 remained close to the meta-model predictions. Without optimization of the operating conditions at part load, the local variations in temperature were of $25 \mathrm{~K}$ and $38 \mathrm{~K}$ with a methane pre-reforming rate of 0.99 and 0.25 . Maximum local values of the variations lower than $13 \mathrm{~K}$ and $10 \mathrm{~K}$ were achieved by optimization. Control consideration related to the measurement of the stack solid temperature will likely be limiting for practical implementation.

(a)

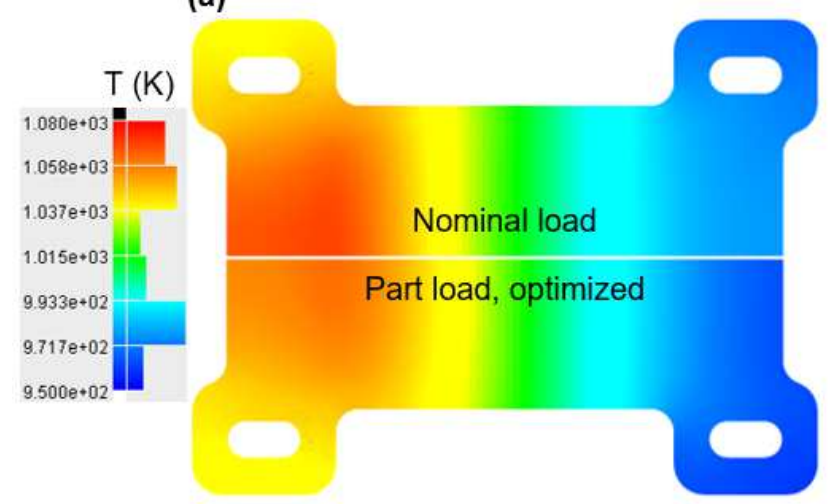

(c)

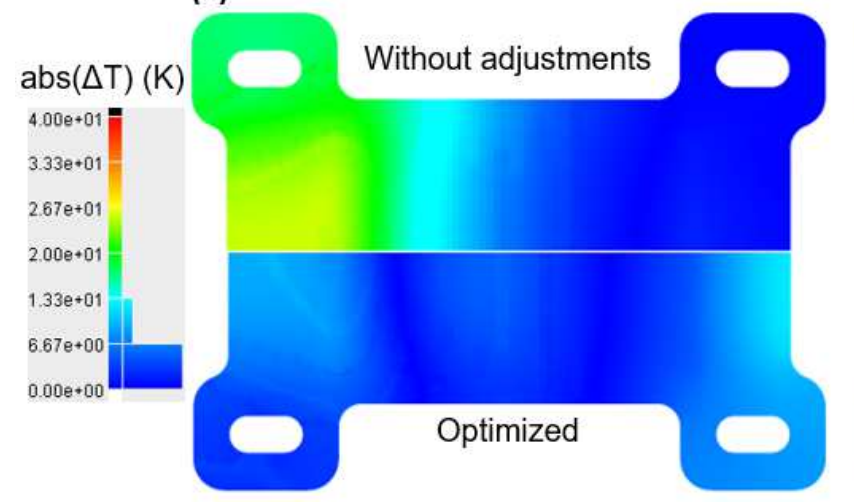

(b)

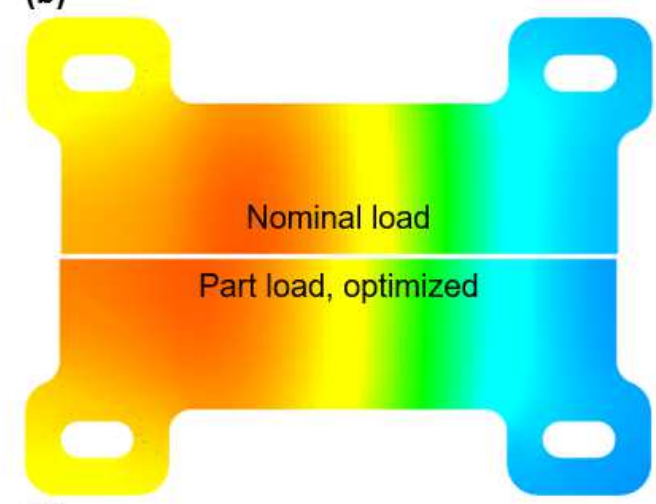

(d)

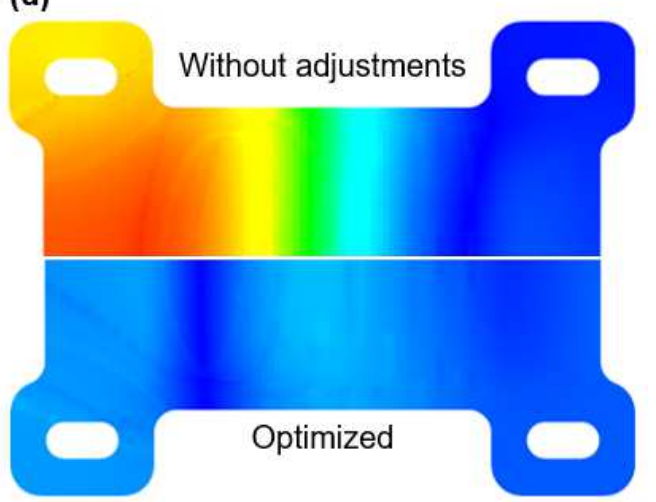

Figure 14. (a-b) Comparison between the solid temperature at nominal load (upper) and part load (lower) with operating conditions at part load optimized with the metamodelbased optimization for $99 \%$ and $25 \%$ of pre-reformed methane. (c-d) Corresponding local temperature difference between nominal and part load, with (lower) and without (upper) optimization.

\section{Conclusions}

An innovative methodology for stack design optimization was shown based on the results of the PROSOFC project. Multi-physics stack models were established and used in a sensitivity analysis which considered the influence of the statistical behavior of 
material, electrochemical and operating parameters. Dedicated material and electrochemical models were experimentally developed to support the multi physics stack modeling. So called meta models were then derived from the multi physics models and automatically operated by the software optiSlang to carry out a sensitivity analysis. Meta models were required to reduce simulation time as the multi physics stack model are too complex to allow a fast sensitivity analysis.

Two examples were selected to showcase the capability and relevance of the methodology. A meta model of the displacement at the inner roller during 4-point bending creep testing was generated from a 3-D finite-element continuum model including intrinsic errors such as the effects of large and 3-D deformation and friction between the sample and the rollers. The parameters of a creep strain hardening constitutive law were identified by optimization. The approach therefore provides improved accuracy and flexibility in terms of analyzed physics, compared to standard post-processing based upon closed form analytical solutions. The developed workflow was successfully tested numerically before application on data from creep experiments on the Ni-YSZ electrode material.

A 3-D meta model of the spatial distribution of temperature in the SOFC stack solid parts was generated from CFD simulations. The accuracy of the meta model proved satisfactory over a window of operating conditions exceeding that of relevance for standard SOFC applications. Therefore, complex 3-D temperature profiles in SOFC stack under operation can be accurately estimated within a few seconds. This new capability was used to minimize local variations of the temperature during discrete switch from nominal to part load. Maximum values of the local changes lower than $13 \mathrm{~K}$ could be achieved by manipulations of the stack operating conditions at part load. This is a significant improvement over standard operation strategies, which typically result in computed variations in the range of $25-40 \mathrm{~K}$.

The developed methodology is a very powerful tool for the identification of those parameters that have an impact on the performance and robustness of the stack design. The parameter analysis can be expanded on the whole lifecycle of the stack and thus include production, conditioning and operation of the stack. This way a comprehensive approach for stack design optimization is provided.

\section{Acknowledgments}

This project has received funding from the Fuel Cell and Hydrogen Joint Undertaking "Seventh Framework Programme of the European Union" under grant agreement No 325278 .

\section{References}

1. T.T. Molla, K. Kwok, and H.L. Frandsen, Int. J. Hydrogen Energy, 41(15), 64336444 (2016).

2. F. Greco, A. Nakajo, Z. Wuillemin, J. Van herle, ECS Trans. 68(1), 1921-1931, 2015. 
3. A. Nakajo, Z. Wuillemin, P. Metzger, S. Diethelm, G. Schiller, J. Van herle, and D. Favrat. J. Electrochem. Soc. 158(9), B1083-B1101, (2011).

4. A. Nakajo, F. Mueller, J. Brouwer, J. Van herle, and D. Favrat. J. Power Sources 216, 449-463, (2012).

5. D.-W. Ni, B. Charlas, K. Kwok, T.T. Molla, P.V. Hendriksen, and H.L. Frandsen, J. Power Sources, 311, 1-12 (2016).

6. G. Pećanac, J. Wei, J. Malzbender, J Power Sources, 327, 629-637 (2016).

7. L.J. Vandeperre, X. Wang, and A. Atkinson, J. Eur. Ceram. Soc., 36(8) 2-6 (2016).

8. X. Wang and A. Atkinson, J. Eur. Ceram. Soc., 35(13) 3713-3720 (2015).

9. J. Wei, G. Pećanac, J. Malzbender, Ceram. Int., 41, 15122-15127 (2015).

10. F. Greco, H.L. Frandsen, A. Nakajo, M.F. Madsen, and J. Van herle, J. Eur. Ceram. Soc., 34(11) 2695-2704 (2014).

11. J. Wei; J. Malzbender, "Steady state creep of Ni-8YSZ substrates for application in solid oxide fuel and electrolysis cells", submitted.

12. T.T. Molla, K. Kwok, and H.L. Frandsen, J. Power Sources, 351, 8-16 (2017).

13. H.L. Frandsen, M. Makowska, C. Chatzichristodoulou, F. Greco, D.W. Ni, D.J. Curran, M. Strobl, L. Theil Kuhn, et al., J. Power Sources, 323, 78-89 (2016).

14. T. T. Molla, F. Greco, K. Kwok, P. Zielke, H. L. Frandsen, ’Development of high temperature mechanical rig for characterizing the viscoplastic properties of alloys used in solid oxide cells", submitted.

15. T.T. Molla, K. Kwok, and H.L. Frandsen, Int. J. Hydrogen Energy, 41(15), 64336444 (2016).

16. K. Kwok, P.S. Jørgensen, and H.L. Frandsen, J. Am. Ceram. Soc., 98(9), 1-8 (2015).

17. T. Osipova, J. Wei, G. Pećanac, J. Malzbender, Ceram. Int., 42, 12932-12936 (2016).

18. J. Wei, G. Pećanac, J. Malzbender, Ceram. Int., 40, 15371-15380 (2014).

19. L. Blum et al. J. Power Sources 196, 7175-7181 (2011).

20. A. Leonide, V. Sonn, A. Weber and E. Ivers-Tiffée, "Evaluation and modeling of the cell resistance in anode-supported solid oxide fuel cells", J. Electrochem. Soc. 155, B36-B41 (2008).

21. M. Schönleber, D. Klotz and E. Ivers-Tiffée, "A Method for Improving the Robustness of linear Kramers-Kronig Validity Tests", Electrochim Acta 131, 2027 (2014).

22. H. Schichlein, A. C. Müller, M. Voigts, A. Krügel and E. Ivers-Tiffée, "Deconvolution of electrochemical impedance spectra for the identification of electrode reaction mechanisms in solid oxide fuel cells", Journal of Applied Electrochemistry 32, 875-882 (2002).

23. A. Leonide, B. Rüger, A. Weber, W. A. Meulenberg and E. Ivers-Tiffée, "Impedance Study of Alternative ( $\mathrm{La}, \mathrm{Sr}) \mathrm{FeO}_{3-\delta}$ and $(\mathrm{La}, \mathrm{Sr})(\mathrm{Co}, \mathrm{Fe}) \mathrm{O}_{3-\delta} \mathrm{MIEC}$ Cathode Compositions", J. Electrochem. Soc. 157, B234-B239 (2010).

24. P. Blennow, J. Hjelm, T. Klemensoe, S. Ramousse, A. Kromp, A. Leonide and A. Weber, "Manufacturing and Characterization of Metal Supported Solid Oxide Fuel Cells", J. Power Sources 196, 7117-7125 (2011).

25. F. Han, R. Mücke, T. van Gestel, A. Leonide, N. H. Menzler, H. P. Buchkremer and D. Stöver, "Novel high-performance solid oxide fuel cells with bulk ionic conductance dominated thin-film electrolytes", J. Power Sources 218, 157-162 (2012). 
26. V. Sonn, A. Leonide and E. Ivers-Tiffée, "Combined Deconvolution and CNLS Fitting Approach Applied on the Impedance Response of Technical Ni/8YSZ Cermet Electrodes", J. Electrochem. Soc. 155, B675-B679 (2008).

27. S. Dierickx, A. Weber and E. Ivers-Tiffée, "Electrochemical Analysis of Sulfur Poisoning in Ni/8YSZ Cermet Anodes", in Nigel Brandon (Ed.), 12th European SOFC \& SOE Forum 2016 NaN, p. B0502 (2016).

28. H. Gerischer, "Wechselstrompolarisation von Elektroden mit einem potentialbestimmenden Schritt beim Gleichgewichtspotential I", Zeitschrift für Physikalische Chemie 198, 286-313 (1951).

29. S. B. Adler, J. A. Lane and B. C. H. Steele, "Electrode kinetics of porous mixedconducting oxygen electrodes", J. Electrochem. Soc. 143, 3554-3564 (1996).

30. L. Almar, J. Szasz, A. Weber and E. Ivers-Tiffée, "Oxygen Transport Kinetics of Mixed Ionic-Electronic Conductors by Coupling Focused Ion Beam Tomography and Electrochemical Impedance Spectroscopy", J. Electrochem. Soc. 164, F289F297 (2017).

31. C. Endler, A. Leonide, A. Weber, F. Tietz and E. Ivers-Tiffée, "Time-Dependent Electrode Performance Changes in Intermediate Temperature Solid Oxide Fuel Cells", J. Electrochem. Soc. 157, B292-B298 (2010).

32. A. Kromp, J. Nielsen, P. Blennow, T. Klemenso and A. Weber, "Break-down of Losses in High Performing Metal-Supported Solid Oxide Fuel Cells", Fuel Cells 13, 598-604 (2013).

33. A. Leonide, S. Hansmann and E. Ivers-Tiffée, "A 0-Dimensional Stationary Model for Anode-Supported Solid Oxide Fuel Cells", ECS Trans. 28, 341-346 (2010).

34. A. Leonide, S. Hansmann, A. Weber and E. Ivers-Tiffée, "Performance simulation of current/voltage-characteristics for SOFC single cell by means of detailed impedance analysis", J. Power Sources 196, 7343-7346 (2011).

35. D. Klotz, A. Leonide, A. Weber and E. Ivers-Tiffée, "Electrochemical model for SOFC and SOEC mode predicting performance and efficiency", International Journal of Hydrogen Energy 39, 20844-20849 (2014).

36. H. Geisler, A. Kromp, A. Weber and E. Ivers-Tiffée, "Stationary FEM Model for Performance Evaluation of Planar Solid Oxide Fuel Cells Connected by Metal Interconnectors", J. Electrochem. Soc. 161, F778-F788 (2014).

37. A. Nakajo, F. Mueller, J. Brouwer, J. Van herle, and D. Favrat. Int. J. Hydrogen Energy 37, 9269-9286, 2012.

38. M. Fardadi, F. Mueller, and F. Jabbari, J. Power Sources, 195(13), 4222-4233, (2010).

39. A. Nakajo, F. Mueller, D. McLarty, J. Brouwer, J. Van herle and D. Favrat. J. Electrochem. Soc. 158(11), B1329-B1340, (2011).

40. FIRE ${ }^{\mathrm{TM}}$ v2017, User Manual, AVL List GmbH, (2017). 